\title{
Compatibility of Diafenthiuron with Selected Agro-Chemicals on Bt Cotton
}

\author{
Bontha Rajasekar* and C.P. Mallapur \\ Department of Agricultural Entomology, University of Agricultural Sciences, \\ Dharwad, India, 580005 \\ *Corresponding author
}

\begin{tabular}{|c|c|}
\hline & A B S T R A C T \\
\hline & \multirow{9}{*}{$\begin{array}{l}\text { Experiments were conducted to assess the emulsion stability revealed that out of four } \\
\text { chemicals tested namely, carbendazim, copper oxychloride, NAA, and } \mathrm{MgSO}_{4} \text { with } \\
\text { diafenthiuron } 50 \mathrm{WP} \text { at } 0.6 \mathrm{~g} \text { litre } \mathrm{e}^{-1} \text {, none of these products produced creaming matter or } \\
\text { sediment, more than } 2.0 \mathrm{ml} \text { at the top or bottom of the } 100 \mathrm{ml} \text { cylinder. The results } \\
\text { confirmed physical stability of these agro-chemicals with diafenthiuron } 50 \mathrm{WP} \text {. A field } \\
\text { trial was conducted to evaluate the bioefficacy of combinations of diafenthiuron } 50 \mathrm{WP} \\
\text { with selected Agro-chemicals, as foliar application on } 90 \text { days old } \mathrm{Bt} \text { cotton against } \\
\text { sucking pests and foliar diseases. The lowest mean population of aphids }(3.28 / 3 \text { leaves), } \\
\text { leafhoppers }\left(3.773 \text { leaves }^{-1}\right) \text {, thrips }\left(1.973 \text { leaves }^{-1}\right) \text {, whiteflies }\left(0.77 / 3 \text { leaves }{ }^{-1}\right) \text { and } \\
\text { mirid bugs }\left(2.00 \text { five squares }{ }^{-1}\right) \text { were recorded in diafenthiuron } 50 \mathrm{WP} @ 0.6 \mathrm{~g}+ \\
\text { carbendazim } 50 \mathrm{WP} @ 1.0 \mathrm{~g} \text { with }(70.33,66.81,89.28,87.78 \text { and } 50.35) \text { high } \% \text { reduction } \\
\text { over control, respectively. Treatments, diafenthiuron in combination with carbendazim } \\
(30.01) \text { and copper oxychloride }(29.36) \text { were recorded high } \% \text { disease over control along } \\
\text { with the alone fungicidal treatments against Alternaria blight and Bacterial blight, } \\
\text { respectively. Diafenthiuron in combination with carbendazim and copper oxychloride were } \\
\text { found to be more effective in reducing the sucking pests population and foliar diseases } \\
\text { incidence, and safer to the three natural enemies (coccinellids, chrysopids and spiders). All } \\
\text { the tested treatments had not caused any phytotoxic symptoms on } 90 \text { days old Bt cotton } \\
\text { crop. }\end{array}$} \\
\hline Keywords & \\
\hline Compatibility, & \\
\hline $\begin{array}{l}\text { Combinations, } \\
\text { Diafenthiuron. }\end{array}$ & \\
\hline Sucking pests, & \\
\hline Diseases and Cotton & \\
\hline Article Info & \\
\hline $\begin{array}{l}\text { Accepted: } \\
26 \text { April } 2017 \\
\text { Available Online: } \\
10 \text { May } 2017\end{array}$ & \\
\hline & \\
\hline
\end{tabular}

\section{Introduction}

Cotton (Gossypium hirsutum L.), popularly known as "the white gold", is an important commercial fibre crop grown under diverse agro-climatic conditions around the world. Introduction of second generation $\mathrm{Bt}$ cotton has given solution to the bollworm complex to the larger extent but at the same time they are susceptible to most of the sucking pests viz., aphid, leafhopper, thrips, whitefly and mirid bug, which occupied major pest status and contributed to lower yields. Apart from this, the diseases like Alternaria leaf spot and Bacterial blight are also posing threat to cotton cultivation. It requires large number of chemicals and sprays for managing different pests. It is often economical and convenient to apply a mixture of two or more pesticides and nutrients when a wide range of pests or maladies are to be managed at a time. This saves time, labour and cost which are the three major but scarce inputs in agricultural systems nowadays (Stanley et al., 2010). Incompatibility may cause loss of effectiveness, poor application and also phytotoxicity. Chemical incompatibility occurs when the material breaks down in to 
different compounds or when the products chemically combine to produce another, which involves deactivation and may result in complete or partial failure. Hence, knowledge on the chemical compatibility is necessary to be familiar with the efficacy of mixed chemicals in managing insect pest and diseases in field condition. In this background, experiments were designed to know the compatibility of a newer insecticide, diafenthiuron 50 WP with other agrochemicals against sucking pests, foliar diseases and natural enemies on Bt cotton.

\section{Materials and Methods}

\section{Emulsion stability test}

Diafenthiuron 50 WP was subjected to physical test for emulsion stability either alone or after mixing with fungicides or fertilizer or growth regulator such as carbendazim, copper oxychloride, NAA, and $\mathrm{MgSO}_{4}$. Emulsion stability test was carried out for diafenthiuron alone and for the combination products as prescribed by Indian Standard specifications (IS, 1973). Standard hard water was prepared by dissolving 0.304 $\mathrm{g}$ anhydrous calcium chloride $\left(\mathrm{CaCl}_{2}\right)$ and $0.139 \mathrm{~g}$ magnesium chloride $\left(\mathrm{MgCl}_{2}\right)$ in one liter of distilled water. This solution had hardness equivalent of $342 \mathrm{ppm}$ calcium carbonate and was used to prepare the insecticide test solutions. To such formulated insecticide suspension $(30 \mathrm{ml}), 30 \mathrm{ml}$ of either of the proposed combination chemical (carbendazim or copper oxychloride or NAA or $\mathrm{MgSO}_{4}$ ) was added separately and transferred to a clean dry graduated cylinder and the volume was made up to $100 \mathrm{ml}$ with standard hard water. The mixture was shaken well and kept in a thermostat at $30 \pm 1^{\circ} \mathrm{C}$ for 1 $\mathrm{h}$ without any disturbance. The observations were taken visually on the formation of creaming matter or sediment not exceeding $2.0 \mathrm{ml}$ at the top or bottom of the $100 \mathrm{ml}$ cylinder, respectively which was considered as the criteria for the compatibility.

\section{Biological compatibility}

A field trial was conducted to evaluate the bioefficacy of combinations of diafenthiuron $50 \mathrm{WP}$ with fungicides or fertilizer or growth regulator, as foliar application on Bt cotton. The experiment was laid out in a Randomized Block Design (RBD) at Main Agricultural Research Station, Dharwad during kharif, 2014-15 season. The experiment consisted of 11 treatments replicated thrice (Table 1). A cotton hybrid, $\mathrm{RCH}-2 \mathrm{Bt}$ susceptible to insect pests and diseases was choosen and raised in plots of $5.40 \times 2.70$ meters with $90 \times 60 \mathrm{~cm}$ row to row and plant to plant spacing. Crop was raised by following package of practices.

For the experiment spraying was carried out using hand operated pneumatic knapsack sprayer with 500 litres of spray fluid ha ${ }^{-1}$ at 90 days after sowing. The population of sucking pests viz., nymphs and adults of aphids, leaf hopper, thrips and whiteflies were recorded from ten randomly selected and tagged plants in each replication. In each plant, three leaves (top, middle and bottom) were considered for observation. Similarly, the counts on mirid bug population on 5 squares per plant were recorded on 5 randomly selected plants. The observations were made prior to spraying, 3, 7 and 14 days after spraying. The insecticide acetamiprid was selected as a standard for further comparison. The observations were recorded on 5 plants on number of fruiting branches plant ${ }^{-1}$ in case of NAA and $\mathrm{MgSO}_{4}$ treatment combinations. In the fungicide combination treatments, the observations were made on diseases like Alternaria leaf spot and Bacterial blight at 0-4 disease rating scale on 5 plants. Then these grades were converted into per cent disease indices (PDI) by using the formula (Sheo Raj, 1988). 


\section{Sum of numerical ratings $\mathrm{x} 100$ \\ Total number of leaves observed $\mathrm{x}$ Maximum disease grade}

The observations were made prior to spraying, 3, 7 and 14 days after spraying. Means of observations 14 days after spray were stated in Table 4. The data obtained from field experiments was analysed in randomized block design (RBD) (Gomez and Gomez, 1984). The mean values were separated using Duncan's Multiple Range Test (DMRT) (Duncan, 1951).

\section{Phytotoxic (plant) compatibility test}

The observations were made at 3, 7 and 14 days after treatment on randomly selected 5 plants in each plot on phytotoxicity at 0-10 scale (Table 2) with following symptoms i.e. injury to leaf tip and leaf surface, wilting, vein clearing, necrosis and epinasty and hyponasty (Rajeshwaran et al., 2004) on 90 days old Bt cotton.

\section{Results and Discussion}

The results on the investigations carried out to study the physical stability in terms of emulsion stability revealed that out of agrochemicals tested namely, carbendazim, copper oxychloride, NAA and $\mathrm{MgSO}_{4}$ with diafenthiuron 50 WP @ $0.6 \mathrm{~g}^{\text {litre }}{ }^{-1}$, none of these products produced creaming matter or sediment, more than $2.0 \mathrm{ml}$ at the top or bottom of the $100 \mathrm{ml}$ cylinder. The results confirmed the physical stability of diafenthiuron 50 WP with selected agrochemicals. The findings of the present study are in agreement with the findings of Stanley et al. (2010) opined that none of the chemicals tested viz., urea, borax, molybdenum, mancozeb, copper oxychloride and carbendazim in combination with diafenthiuron 50 WP at $1.6 \mathrm{~g}$ litre $^{-1}$, produced creaming matter or sediment, more than 2.0 $\mathrm{ml}$ at the top or bottom of the $100 \mathrm{ml}$ measuring cylinder by means of physical emulsion stability test.

The experimental results of investigations carried out on the evaluation of biological compatibility of diafenthiuron with fungicides or fertilizer or growth regulator was assessed against sucking pests and foliar diseases and the results are as follows.

\section{Sucking pests}

The results of the present investigation revealed that the lowest mean aphid population (14 days after spray) was recorded

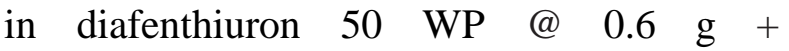
carbendazim 50WP @ 1 g (3.28 aphids 3 leaves $^{-1}$ ) with $70.73 \%$ reduction over untreated check. Similar trend was noticed in other treatments, diafenthiuron 50 WP alone and its combinations with copper oxychloride 50 WP @ 2.0 g, NAA 20 ppm and $\mathrm{MgSO}_{4} @$ $10 \mathrm{~g}$ (3.57 to 3.81 aphids 3 leaves $^{-1}$ with 68.17 to $66.02 \%$ reduction over control). The university check acetamiprid 20 SP @ $0.2 \mathrm{~g}$ was shown the mean population (5.28 aphids 3 leaves $^{-1}$ ) with $52.93 \%$ reduction over untreated check. The lowest mean population of leafhopper was recorded in diafenthiuron 50WP@0.6 g + carbendazim 50WP @1.0 g (3.77 leafhoppers 3 leaves $^{-1}$ ) with $66.81 \%$ reduction over untreated check. Sequentially, the remaining treatments i.e., diafenthiuron 50 WP alone and its combinations, and acetamiprid $20 \mathrm{SP}$ were recorded mean leafhopper population of 3.87 to 4.98 leafhoppers 3 leaves $^{-1}$ with 65.93 to $56.09 \%$ reduction over untreated check. The treatment diafenthiuron 50 WP @0.6 g + carbendazim 50WP@1.0 g was noticed lowest mean thrips population (1.97 thrips 3 leaves $^{-1}$ ) with $89.28 \%$ reduction over untreated check, followed by diafenthiuron 50 WP alone and its combinations, which were significantly on 
par with each other in reducing the number of thrips population. The university check acetamiprid 20 SP @ $0.2 \mathrm{~g}$ was shown the mean population (4.89 thrips 3 leaves $^{-1}$ ) with $73.41 \%$ reduction over untreated check. The number of mean whiteflies population per three leaves and \% reduction over control recorded in following treatments, diafenthiuron 50 WP @ $0.6 \mathrm{~g}+$ carbendazim 50WP@1.0 g (0.77 3 leaves $^{-1}$ and 87.78) followed by diafenthiuron 50 WP alone @ 0.6 g (0.88 3 leaves $^{-1}$ and 86.14), diafenthiuron 50 WP @ 0.6 g + copper oxychloride 50WP @ 2g (0.92 3 leaves $^{-1}$ and 85.43), diafenthiuron 50 WP @ 0.6 g + NAA @ 20 ppm (0.95 3 leaves $^{-1}$ and 84.96) and diafenthiuron 50 WP @ $0.6 \mathrm{~g}+\mathrm{MgSO}_{4} @ 10 \mathrm{~g}$ (1.00 3 leaves $^{-1}$ and 84.19) respectively (Table 4). The university check acetamiprid 20 SP @ $0.2 \mathrm{~g}$ was shown the mean population (2.62 whiteflies 3 leaves $^{-1}$ ) with $58.49 \%$ reduction over untreated check. The lowest mean population of mirid bugs were recorded in diafenthiuron 50 WP @ $0.6 \mathrm{~g}+$ carbendazim 50WP@1.0 g (2.00 mirid bugs five squares $\left.{ }^{1}\right)$ with $50.35 \%$ reduction over untreated check. Consecutively, the remaining treatments i.e., diafenthiuron $50 \mathrm{WP}$ alone and its combinations, and acetamiprid $20 \mathrm{SP}$ were recorded mean mirid bug population of 2.01 to 2.32 mirid bugs five squares ${ }^{-1}$ with 50.06 to $42.36 \%$ reduction over untreated check. Whereas, all the non-insecticidal treatments were shown poor results in reducing the all sucking pest population. Diafenthiuron is showing synergistic action, when it combined with carbendazim in reducing sucking pest population in cotton field. The findings of the present study are in agreement with the findings of Stanley et al. (2010) revealed that diafenthiuron alone has recorded $52.77 \%$ reduction while diafenthiuron + carbendazim recorded the maximum reduction of $55.80 \%$ against cardamom thrips. Further, the results are in agreement with the findings of Bharpoda et al. (2014) who noticed that difenthiuron 50 WP $(0.05 \%)$ recorded significant lower population of the leafhopper (1.66 leaf $\left.{ }^{-1}\right)$ and whiteflies (1.67 leaf $\left.{ }^{-1}\right)$ compared to all other treatments.

\section{Foliar diseases}

The lowest per cent disease index of bacterial blight was recorded in copper oxychloride @ $2 \mathrm{~g}$ (20.94) followed by diafenthiuron $50 \mathrm{WP}$ @0.6 g + copper oxychloride 50WP @ $2 \mathrm{~g}$ (21.27), carbendazim 50WP @1.0 g (22.53) and diafenthiuron 50 $\quad$ WP $@ 0.6$ g + carbendazim 50WP @ 1.0 g (23.17) with 30.46, 29.36, 25.19 and 23.06\% disease over control, respectively. The treatments, diafenthiuron 50 WP @ $0.6 \mathrm{~g}+$ carbendazim 50WP @ $1 \mathrm{~g}$ (20.28 and 30.01) followed by carbendazim 50WP @ $1.0 \mathrm{~g}$ (19.45 and 32.85), copper oxychloride 50WP @ 2g (20.18 and 30.33) and diafenthiuron 50 WP @ $0.6 \mathrm{~g}+$ copper oxychloride 50WP @ $2 \mathrm{~g}$ (21.34 and 26.34) were recorded low mean \% disease index and high percent disease over control respectively, in reducing the alternaria blight (Table 4). Whereas, the treatments, doesn't have fungicide or its combination shown poor results in reducing the bacterial blight and alternaria blight. The results are in agreement with the studies of Jagtap et al., (2012) reported that low disease incidence of bacterial blight was recorded in treatment copper oxychloride $0.25 \%+$ streptocycline $100 \mathrm{ppm}$.

\section{Natural enemies}

The observations in treatments like diafenthiuron $50 \mathrm{WP}$, carbendazim $50 \mathrm{WP}$, copper oxychloride $50 \mathrm{WP}, \mathrm{NAA}, \mathrm{MgSO}_{4}$ and acetamiprid alone and combinations were revealed that there was non-significant difference among the treatments, which were statistically on par with each other and found to be safer towards three natural enemies viz., 
coccinellids, chrysopids and spiders (Table 4). The results are in line with the reports of Bharpoda et al., (2014) stated that diafenthiuron 50 WP @ 0.05\% was found more effective against pest population and safer to the natural enemies viz., Chrysopids, spiders and coccinellids.

\section{Yield and economics}

The highest fruiting branches plant $^{-1}$ mean values were recorded in diafenthiuron $50 \mathrm{WP}$ @0.6 g + NAA @ 20 ppm (23.53) followed by NAA @ 20 ppm (23.49), diafenthiuron 50 WP @0.6 g + $\mathrm{MgSO}_{4} @ 10 \mathrm{~g}$ (22.35) and $\mathrm{MgSO}_{4} @ 10 \mathrm{~g}$ (22.34) with 13.43, 13.28, 8.87 and $8.83 \%$ increase over control, respectively (Table 4). Whereas, the treatments, doesn't have fertilizer or growth regulator or its combination shown poor results in increasing the fruiting branches plant $^{-1}$. The results of present study are in line with the investigations of Rajendran et al. (2005) reported that foliar application of NAA $40 \mathrm{ppm}$ recorded higher number of sympodial branches plant ${ }^{-1}$, bolls plant ${ }^{-1}$ and seed cotton yield. Foliar application of $1 \%$
$\mathrm{MgSO}_{4}$ during flowering to boll development stage significantly resulted in higher seed cotton yield (2066 Kg ha ${ }^{-1}$ ) (Basavanneppa et al., 2009). In all the treatments, no phytotoxicity symptom was observed. The highest yield per hectare was recorded in diafenthiuron 50 WP @0.6 g + carbendazim 50WP @ $1.0 \mathrm{~g} \quad\left(19.07 \mathrm{q} \quad \mathrm{ha}^{-1}\right)$ and diafenthiuron 50 WP @ $0.6 \mathrm{~g}+$ copper oxychloride 50WP @ 2g (18.18 q ha $\left.{ }^{-1}\right)$ found to be significantly superior over rest of the treatments but were on par among themselves, however, these treatments recorded 1.01 and 0.91 of benefit cost ratio's, respectively, which were higher than the treatment acetamiprid 20 SP @ 0.2 g (13.48 q ha $\left.{ }^{-1}\right)$ recorded 0.54 benefit cost ratio. Treatments, diafenthiuron when sprayed in combination with carbendazim and copper oxychloride were found to be more effective against aphids, leafhoppers, thrips, whiteflies, mirid bugs, alternaria blight and bacterial blight with higher pest reduction values over control than when used alone or their combinations with NAA and $\mathrm{MgSO}_{4}$. All the treatments were found safer to the natural enemies.

Table.1 Treatments details

\begin{tabular}{|c|l|c|}
\hline Sl. no & \multicolumn{1}{|c|}{ Treatments } & $\begin{array}{c}\text { Dosage } \\
\text { (g or ml) } \text { Litre }^{-1}\end{array}$ \\
\hline 1 & Diafenthiuron 50 WP & $0.6 \mathrm{~g}$ \\
\hline 2 & $\begin{array}{l}\text { Diafenthiuron 50 WP + Carbendazim 50 } \\
\text { WP }\end{array}$ & $0.6 \mathrm{~g}+1 \mathrm{~g}$ \\
\hline 3 & $\begin{array}{l}\text { Diafenthiuron 50 WP + Copper } \\
\text { oxychloride 50 WP }\end{array}$ & $0.6 \mathrm{~g}+2 \mathrm{~g}$ \\
\hline 4 & Diafenthiuron 50 WP + NAA & $0.6 \mathrm{~g}+20 \mathrm{ppm}$ \\
\hline 5 & Diafenthiuron 50 WP + MgSO & $0.6 \mathrm{~g}+10 \mathrm{~g}$ \\
\hline 6 & Acetamaprid 20SP & $0.2 \mathrm{~g}$ \\
\hline 7 & Carbendazim 50 WP & $1.0 \mathrm{~g}$ \\
\hline 8 & Copper oxychloride 50 WP & $2 \mathrm{~g}$ \\
\hline 9 & NAA & $20 \mathrm{ppm}$ \\
\hline 10 & MgSO $_{4}$ & $10 \mathrm{~g}$ \\
\hline 11 & Untreated check & - \\
\hline
\end{tabular}


Table.4 Biological compatibility of diafenthiuron with selected agrochemicals against sucking pests,

foliar diseases and natural enemies in cotton

\begin{tabular}{|c|c|c|c|c|c|c|c|c|c|c|c|c|c|c|}
\hline \multirow{2}{*}{ Treatments } & \multicolumn{2}{|c|}{$\begin{array}{l}\text { Aphids } \\
3 \text { leaves }^{-1}\end{array}$} & \multicolumn{2}{|c|}{$\begin{array}{c}\text { Leafhoppers } \\
3 \text { leaves }^{-1}\end{array}$} & \multicolumn{2}{|c|}{$\begin{array}{c}\text { Thrips } \\
3 \text { leaves }^{-1}\end{array}$} & \multicolumn{2}{|c|}{$\begin{array}{l}\text { Whiteflies } \\
3 \text { leaves }^{-1}\end{array}$} & \multicolumn{2}{|c|}{$\begin{array}{l}\text { mirid bugs } \\
5 \text { squares }^{-1}\end{array}$} & \multicolumn{2}{|c|}{ Bacterial blight } & \multicolumn{2}{|c|}{ Alternaria blight } \\
\hline & Mean & $\% \mathrm{RC}$ & Mean & $\% \mathrm{RC}$ & Mean & $\% \mathrm{RC}$ & Mean & $\% \mathrm{RC}$ & Mean & $\% \mathrm{RC}$ & $\% \mathrm{DI}$ & $\% \mathrm{DC}$ & $\% \mathrm{DI}$ & $\% \mathrm{DC}$ \\
\hline Diafenthiuron $50 \mathrm{WP}$ & $\begin{array}{c}3.57 \\
(2.02) \mathrm{b}\end{array}$ & 68.17 & $\begin{array}{c}3.87 \\
(2.09) \mathrm{b}\end{array}$ & 65.93 & $\begin{array}{c}2.15 \\
(1.63) \mathrm{c}\end{array}$ & 88.33 & $\begin{array}{c}0.88 \\
(1.17) \mathrm{c}\end{array}$ & 86.14 & $\begin{array}{c}2.01 \\
(1.58) \mathrm{b}\end{array}$ & 50.06 & $\begin{array}{c}28.73 \\
(32.40) \mathrm{ab}\end{array}$ & 4.62 & $\begin{array}{c}27.73 \\
(31.76) \mathrm{ab}\end{array}$ & 4.28 \\
\hline $\begin{array}{c}\text { Diafenthiuron } 50 \mathrm{WP}+ \\
\text { Carbendazim 50 WP }\end{array}$ & $\begin{array}{c}3.28 \\
(1.95) \mathrm{b}\end{array}$ & 70.73 & $\begin{array}{c}3.77 \\
(2.07) \mathrm{b}\end{array}$ & 66.81 & $\begin{array}{c}1.97 \\
(1.57) \mathrm{c}\end{array}$ & 89.28 & $\begin{array}{c}0.77 \\
(1.13) \mathrm{c}\end{array}$ & 87.78 & $\begin{array}{c}2.00 \\
(1.58) \mathrm{b}\end{array}$ & 50.35 & $\begin{array}{c}23.17 \\
(28.76) \mathrm{ab}\end{array}$ & 23.06 & $\begin{array}{c}20.28 \\
(26.75) \mathrm{cd}\end{array}$ & 30.01 \\
\hline $\begin{array}{c}\text { Diafenthiuron } 50 \mathrm{WP}+ \\
\text { Copper oxychloride } 50 \mathrm{WP}\end{array}$ & $\begin{array}{c}3.70 \\
(2.05) \mathrm{b}\end{array}$ & 67.06 & $\begin{array}{c}4.61 \\
(2.26) \mathrm{b}\end{array}$ & 59.43 & $\begin{array}{c}2.36 \\
(1.69) \mathrm{c}\end{array}$ & 87.14 & $\begin{array}{c}0.92 \\
(1.19) \mathrm{c}\end{array}$ & 85.43 & $\begin{array}{c}2.01 \\
(1.58) \mathrm{b}\end{array}$ & 50.04 & $\begin{array}{c}21.27 \\
(27.46) \mathrm{b}\end{array}$ & 29.36 & $\begin{array}{c}21.34 \\
(27.50) \mathrm{bcd}\end{array}$ & 26.34 \\
\hline Diafenthiuron $50 \mathrm{WP}+\mathrm{NAA}$ & $\begin{array}{c}3.76 \\
(2.06)^{\mathrm{b}} \\
\end{array}$ & 66.51 & $\begin{array}{c}4.65 \\
(2.27)^{\mathrm{b}}\end{array}$ & 59.03 & $\begin{array}{c}2.49 \\
(1.73) \mathrm{c}\end{array}$ & 86.48 & $\begin{array}{c}0.95 \\
(1.20) \mathrm{c} \\
\end{array}$ & 84.96 & $\begin{array}{c}2.02 \\
(1.59) \mathrm{b} \\
\end{array}$ & 49.94 & $\begin{array}{c}27.69 \\
(31.74) \mathrm{ab}\end{array}$ & 8.05 & $\begin{array}{c}26.75 \\
(31.13) \mathrm{abc}\end{array}$ & 7.65 \\
\hline Diafenthiuron $50 \mathrm{WP}+\mathrm{MgSO}_{4}$ & $\begin{array}{c}3.81 \\
(2.08) \mathrm{b}\end{array}$ & 66.02 & $\begin{array}{c}4.98 \\
(2.34)^{\mathrm{b}}\end{array}$ & 56.09 & $\begin{array}{c}2.59 \\
(1.76) \mathrm{c}\end{array}$ & 85.91 & $\begin{array}{c}1.00 \\
(1.22) \mathrm{c}\end{array}$ & 84.19 & $\begin{array}{c}2.03 \\
(1.59) \mathrm{b}\end{array}$ & 49.48 & $\begin{array}{c}27.82 \\
(31.82) \mathrm{ab}\end{array}$ & 7.64 & $\begin{array}{c}26.87 \\
(31.21) \mathrm{abc}\end{array}$ & 7.27 \\
\hline Acetamaprid 20SP & $\begin{array}{c}5.28 \\
(2.40) \mathrm{b}\end{array}$ & 52.93 & $\begin{array}{c}4.90 \\
(2.32) \mathrm{b}\end{array}$ & 56.83 & $\begin{array}{c}4.89 \\
(2.32) \mathrm{b}\end{array}$ & 73.41 & $\begin{array}{c}2.62 \\
(1.77) \mathrm{b}\end{array}$ & 58.49 & $\begin{array}{c}2.32 \\
(1.68) \mathrm{b}\end{array}$ & 42.36 & $\begin{array}{c}27.59 \\
(31.67) \mathrm{ab}\end{array}$ & 8.41 & $\begin{array}{c}27.36 \\
(31.53) \mathrm{abc}\end{array}$ & 5.55 \\
\hline Carbendazim 50WP & $\begin{array}{c}9.79 \\
(3.21) \mathrm{a}\end{array}$ & 12.75 & $\begin{array}{c}9.23 \\
(3.12) \mathrm{a} \\
\end{array}$ & 18.72 & $\begin{array}{l}17.55 \\
(4.25) \mathrm{a}\end{array}$ & 4.51 & $\begin{array}{c}5.81 \\
(2.51) \mathrm{a}\end{array}$ & 8.00 & $\begin{array}{c}3.81 \\
(2.08) \mathrm{a}\end{array}$ & 5.42 & $\begin{array}{c}22.53 \\
(28.33) \mathrm{ab}\end{array}$ & 25.19 & $\begin{array}{c}19.45 \\
(26.16) \mathrm{d}\end{array}$ & 32.85 \\
\hline Copper oxychloride 50WP & $\begin{array}{c}10.06 \\
(3.25) \mathrm{a} \\
\end{array}$ & 10.29 & $\begin{array}{r}9.49 \\
(3.16) \mathrm{a} \\
\end{array}$ & 16.37 & $\begin{array}{l}17.88 \\
(4.29) \mathrm{a} \\
\end{array}$ & 2.69 & $\begin{array}{c}5.94 \\
(2.54) \mathrm{a} \\
\end{array}$ & 6.02 & $\begin{array}{c}3.88 \\
(2.09) \mathrm{a} \\
\end{array}$ & 3.51 & $\begin{array}{c}20.94 \\
(27.22) \mathrm{b} \\
\end{array}$ & 30.46 & $\begin{array}{c}20.18 \\
(26.69) \mathrm{cd} \\
\end{array}$ & 30.33 \\
\hline NAA & $\begin{array}{c}10.18 \\
(3.27) \mathrm{a}\end{array}$ & 9.29 & $\begin{array}{c}9.53 \\
(3.17) \mathrm{a}\end{array}$ & 16.01 & $\begin{array}{c}17.92 \\
(4.29) \mathrm{a}\end{array}$ & 2.50 & $\begin{array}{c}6.09 \\
(2.57) \mathrm{a}\end{array}$ & 3.56 & $\begin{array}{c}3.95 \\
(2.11) \mathrm{a}\end{array}$ & 1.96 & $\begin{array}{c}27.76 \\
(31.78) \mathrm{ab}\end{array}$ & 7.83 & $\begin{array}{c}27.28 \\
(31.47) \mathrm{abc}\end{array}$ & 5.85 \\
\hline $\mathrm{MgSO}_{4}$ & $\begin{array}{c}10.28 \\
(3.28) \mathrm{a} \\
\end{array}$ & 8.37 & $\begin{array}{c}9.52 \\
(3.16) \mathrm{a} \\
\end{array}$ & 16.15 & $\begin{array}{c}18.11 \\
(4.31) \mathrm{a} \\
\end{array}$ & 1.48 & $\begin{array}{c}6.21 \\
(2.59) \mathrm{a} \\
\end{array}$ & 1.74 & $\begin{array}{c}4.01 \\
(2.12) \mathrm{a} \\
\end{array}$ & 0.49 & $\begin{array}{c}28.35 \\
(32.16) \mathrm{ab} \\
\end{array}$ & 5.88 & $\begin{array}{c}27.45 \\
(31.58) \mathrm{abc}\end{array}$ & 5.26 \\
\hline Untreated check & $\begin{array}{c}11.22 \\
(3.42) \mathrm{a}\end{array}$ & 0.00 & $\begin{array}{c}11.35 \\
(3.44) \mathrm{a} \\
\end{array}$ & 0.00 & $\begin{array}{c}18.38 \\
(4.34) \mathrm{a} \\
\end{array}$ & 0.00 & $\begin{array}{c}6.32 \\
(2.61) \mathrm{a}\end{array}$ & 0.00 & $\begin{array}{c}4.02 \\
(2.13) \mathrm{a}\end{array}$ & 0.00 & $\begin{array}{c}30.12 \\
(33.27) \mathrm{a}\end{array}$ & 0.00 & $\begin{array}{c}28.97 \\
(32.55) \mathrm{a}\end{array}$ & 0.00 \\
\hline S. Em \pm & 0.15 & & 0.15 & & 0.20 & & 0.15 & & 0.12 & & 1.72 & & 1.48 & \\
\hline $\mathrm{CD}(0.05)$ & 0.47 & & 0.47 & & 0.62 & & 0.47 & & 0.39 & & 5.43 & & 4.65 & \\
\hline $\mathrm{CV}(\%)$ & 9.71 & & 9.57 & & 11.66 & & 13.73 & & 11.65 & & 9.75 & & 9.57 & \\
\hline
\end{tabular}


Table 4 contd.....

\begin{tabular}{|c|c|c|c|c|c|c|c|c|c|}
\hline \multirow{2}{*}{ Treatments } & \multirow{2}{*}{$\begin{array}{c}\begin{array}{c}\text { Coccinellids } \\
5 \text { plants }^{-1}\end{array} \\
\text { Mean }\end{array}$} & \multirow{2}{*}{$\begin{array}{c}\text { Chrysopids } \\
5 \text { plants }^{-1} \\
\text { Mean }\end{array}$} & \multirow{2}{*}{$\begin{array}{c}\begin{array}{c}\text { Spiders } \\
5 \text { plants }^{-1}\end{array} \\
\text { Mean }\end{array}$} & \multicolumn{2}{|c|}{ Fruiting branches plant $^{-1}$} & \multirow{2}{*}{$\begin{array}{l}\text { Yield } \\
\left(\mathbf{Q} \mathbf{h a}^{-1}\right)\end{array}$} & \multirow{2}{*}{$\begin{array}{l}\text { Cost of } \\
\text { cultivation* }\end{array}$} & \multirow{2}{*}{$\begin{array}{l}\text { Net } \\
\text { returns }\end{array}$} & \multirow{2}{*}{$\begin{array}{l}\text { B:C } \\
\text { Ratio }\end{array}$} \\
\hline & & & & Mean & $\% \mathrm{IC}$ & & & & \\
\hline Diafenthiuron $50 \mathrm{WP}$ & $\begin{array}{c}1.53 \\
(1.42)^{\mathrm{a}} \\
\end{array}$ & $\begin{array}{c}1.14 \\
(1.28)^{\mathrm{a}} \\
\end{array}$ & $\begin{array}{c}1.49 \\
(1.41)^{\mathrm{a}} \\
\end{array}$ & 20.44 & 0.34 & 15.36 & 37780.00 & 25210.40 & 0.67 \\
\hline $\begin{array}{c}\text { Diafenthiuron } 50 \mathrm{WP}+ \\
\text { Carbendazim 50 WP } \\
\end{array}$ & $\begin{array}{c}1.52 \\
(1.42)^{\mathrm{a}} \\
\end{array}$ & $\begin{array}{c}1.15 \\
(1.28)^{\mathrm{a}} \\
\end{array}$ & $\begin{array}{c}1.49 \\
(1.41)^{\mathrm{a}} \\
\end{array}$ & 20.39 & 0.09 & 19.07 & 38830.00 & 39345.58 & 1.01 \\
\hline $\begin{array}{c}\text { Diafenthiuron 50 WP + } \\
\text { Copper oxychloride } 50 \mathrm{WP}\end{array}$ & $\begin{array}{c}1.53 \\
(1.43)^{\mathrm{a}}\end{array}$ & $\begin{array}{c}1.16 \\
(1.29)^{\mathrm{a}}\end{array}$ & $\begin{array}{c}1.51 \\
(1.42)^{\mathrm{a}}\end{array}$ & 20.39 & 0.11 & 18.18 & 38952.00 & 35567.89 & 0.91 \\
\hline Diafenthiuron $50 \mathrm{WP}+\mathrm{NAA}$ & $\begin{array}{c}1.53 \\
(1.42)^{\mathrm{a}} \\
\end{array}$ & $\begin{array}{c}1.16 \\
(1.29)^{\mathrm{a}} \\
\end{array}$ & $\begin{array}{c}1.52 \\
(1.42)^{\mathrm{a}} \\
\end{array}$ & 23.53 & 13.43 & 15.84 & 38153.50 & 26805.35 & 0.70 \\
\hline Diafenthiuron $50 \mathrm{WP}+\mathrm{MgSO}_{4}$ & $\begin{array}{c}1.53 \\
(1.42)^{\mathrm{a}} \\
\end{array}$ & $\begin{array}{c}1.15 \\
(1.29)^{\mathrm{a}}\end{array}$ & $\begin{array}{c}1.54 \\
(1.43)^{\mathrm{a}}\end{array}$ & 22.35 & 8.87 & 15.57 & 38520.00 & 25314.02 & 0.66 \\
\hline Acetamaprid 20SP & $\begin{array}{c}1.62 \\
(1.46)^{\mathrm{a}} \\
\end{array}$ & $\begin{array}{c}1.20 \\
(1.30)^{\mathrm{a}} \\
\end{array}$ & $\begin{array}{c}1.61 \\
(1.45)^{\mathrm{a}} \\
\end{array}$ & 20.43 & 0.31 & 13.48 & 35860.00 & 19397.20 & 0.54 \\
\hline Carbendazim 50WP & $\begin{array}{c}1.70 \\
(1.48)^{\mathrm{a}} \\
\end{array}$ & $\begin{array}{c}1.21 \\
(1.31)^{\mathrm{a}} \\
\end{array}$ & $\begin{array}{c}1.81 \\
(1.52)^{\mathrm{a}} \\
\end{array}$ & 20.47 & 0.49 & 10.36 & 36550.00 & 5912.28 & 0.16 \\
\hline Copper oxychloride 50WP & $\begin{array}{c}1.71 \\
(1.49)^{\mathrm{a}} \\
\end{array}$ & $\begin{array}{c}1.23 \\
(1.32)^{\mathrm{a}} \\
\end{array}$ & $\begin{array}{c}1.82 \\
(1.52)^{\mathrm{a}} \\
\end{array}$ & 20.51 & 0.70 & 9.95 & 36672.00 & 4103.03 & 0.11 \\
\hline NAA & $\begin{array}{c}1.69 \\
(1.48)^{\mathrm{a}}\end{array}$ & $\begin{array}{c}1.24 \\
(1.32)^{\mathrm{a}}\end{array}$ & $\begin{array}{c}1.83 \\
(1.53)^{\mathrm{a}}\end{array}$ & 23.49 & 13.28 & 9.40 & 35873.50 & 2651.88 & 0.07 \\
\hline $\mathrm{MgSO}_{4}$ & $\begin{array}{c}1.72 \\
(1.49)^{\mathrm{a}} \\
\end{array}$ & $\begin{array}{c}1.22 \\
(1.31)^{\mathrm{a}} \\
\end{array}$ & $\begin{array}{c}1.82 \\
(1.52)^{\mathrm{a}} \\
\end{array}$ & 22.34 & 8.83 & 8.92 & 36240.00 & 316.93 & 0.01 \\
\hline Untreated check & $\begin{array}{c}1.77 \\
(1.51)^{\mathrm{a}} \\
\end{array}$ & $\begin{array}{c}1.30 \\
(1.34)^{\mathrm{a}} \\
\end{array}$ & $\begin{array}{c}1.84 \\
(1.53)^{\mathrm{a}} \\
\end{array}$ & 20.37 & 0.00 & 8.57 & 35000.00 & 150.89 & 0.00 \\
\hline S. Em \pm & 0.10 & 0.09 & 0.09 & - & - & - & - & - & - \\
\hline $\mathrm{CD}(0.05)$ & NS & NS & $\mathbf{N S}$ & - & - & - & - & - & - \\
\hline CV(\%) & 11.71 & 10.57 & 9.42 & - & - & - & - & - & - \\
\hline
\end{tabular}

Mean = Mean of observations 14 days after spray; $\%$ RC = \% Reduction Over Control; $\%$ DI = \% Disease Index; $\%$ DC = $\%$ Disease Control; $\%$ IC = $\%$ Increase over Control; Figures in the parenthesis are $\sqrt{\mathrm{x}}+0.5$ transformed values. Means followed by same letter do not differ significantly by DMRT (P = 0.05); NS = Non Significant; Cost of cultivation: *-Including plant protection measures; Market price of cotton: 4,100/q; diafenthiuron $\square$ 950/250g; carbendazim $\square$ 105/100 g; copper oxychloride $\square$ 293/500 g; NAA $\square$ 83/100 ml; $\mathrm{MgSO}_{4} \square$ 74/1 Kg and acetamiprid $\square$ 180/100 g, and dosage @ 0.6 g, 1.0 g, 2.0 g, 20 ppm (0.45 ml), 10 g and 0.2 g per litre, respectively. 
Table.2 Phytotoxicity at $0-10$ scale

\begin{tabular}{|c|c|c|c|}
\hline Scale & $\begin{array}{c}\text { Per cent } \\
\text { phytotoxicity }\end{array}$ & Scale & $\begin{array}{c}\text { Per cent } \\
\text { phytotoxicity }\end{array}$ \\
\hline 0 & No phytotoxicity & 6 & $51-60$ \\
1 & $1-10$ & 7 & $61-70$ \\
2 & $11-20$ & 8 & $71-80$ \\
3 & $21-30$ & 9 & $81-90$ \\
4 & $31-40$ & 10 & $91-100$ \\
5 & $41-50$ & & \\
\hline
\end{tabular}

Table.3 Phytotoxic compatibility of diafenthiuron 50 WP with selected agro-chemicals on cotton

\begin{tabular}{|c|l|c|c|c|c|c|c|}
\hline \multirow{2}{*}{$\begin{array}{c}\text { Treatment } \\
\mathbf{S}\end{array}$} & \multicolumn{1}{|c|}{ Mixture } & \multicolumn{5}{|c|}{ Phytotoxicity ratings } \\
\cline { 3 - 7 } & & $\begin{array}{c}\text { Leaf tip } \\
\text { injury }\end{array}$ & $\begin{array}{c}\text { Wiltin } \\
\text { g }\end{array}$ & $\begin{array}{c}\text { Vein } \\
\text { clearin } \\
\mathbf{g}\end{array}$ & $\begin{array}{c}\text { Necrosi } \\
\mathbf{s}\end{array}$ & $\begin{array}{c}\text { Epinast } \\
\mathbf{y}\end{array}$ & $\begin{array}{c}\text { Hyponast } \\
\mathbf{y}\end{array}$ \\
\hline $\mathrm{T}_{1}$ & Diafenthiuron 50 WP & 0 & 0 & 0 & 0 & 0 & 0 \\
$\mathrm{~T}_{2}$ & Diafenthiuron 50 WP + Carbendazim 50 WP & 0 & 0 & 0 & 0 & 0 & 0 \\
$\mathrm{~T}_{3}$ & Diafenthiuron 50 WP + Copper oxychloride 50 & 0 & 0 & 0 & 0 & 0 & 0 \\
& WP & 0 & 0 & 0 & 0 & 0 & 0 \\
$\mathrm{~T}_{4}$ & Diafenthiuron 50 WP + NAA & 0 & 0 & 0 & 0 & 0 & 0 \\
$\mathrm{~T}_{5}$ & Diafenthiuron 50 WP + MgSO & 0 & 0 & 0 & 0 & 0 & 0 \\
$\mathrm{~T}_{6}$ & Acetamiprid 20 SP & 0 & 0 & 0 & 0 & 0 & 0 \\
$\mathrm{~T}_{7}$ & Carbendazim 50 WP & 0 & 0 & 0 & 0 & 0 & 0 \\
$\mathrm{~T}_{8}$ & Copper oxychloride 50 WP & 0 & 0 & 0 & 0 & 0 & 0 \\
$\mathrm{~T}_{9}$ & NAA & 0 & 0 & 0 & 0 & 0 & 0 \\
$\mathrm{~T}_{10}$ & MgSO & 0 & 0 & 0 & 0 & 0 & 0 \\
$\mathrm{~T}_{11}$ & Untreated check & 0 & 0 \\
\hline
\end{tabular}

Results on the investigations conducted to find out the phytotoxic effects of these combination products on $\mathrm{RCH}-2 \mathrm{Bt}$ cotton revealed that diafenthiuron $50 \mathrm{WP}$ at @ $0.6 \mathrm{~g}$ litre $^{-1}$ with the above agro-chemicals at recommended dose had not caused any phytotoxic symptoms such as injury to leaf tip and leaf surface, wilting, vein clearing, necrosis, epinasty and hyponasty on 90 days old cotton crop (Table 3). The results of present study are in line with the investigations of Stanley et al., (2010) revealed that diafenthiuron alone and its combinations with carbendazim, $\mathrm{MgSO}_{4}$ and molybdenum did not cause any phototoxic symptoms such as injury to leaf tip and leaf surface, wilting, vein clearing, necrosis, epinasty and hyponasty in cardamom. It might be the first report in studying the compatibility of diafenthiuron $50 \mathrm{WP}$ with selected agro-chemicals on Bt cotton in India.

In conclusion, the results proved that all the test treatments were physically, biologically and phytotoxically compatible with each other in $\mathrm{Bt}$ cotton. The treatments, diafenthiuron in combination with carbendazim was found to be more effective in reducing the sucking pests population like aphids, leafhoppers, thrips, whiteflies and one foliar disease, Alternaria Blight but less effective in case of mirid bugs. Diafenthiuron in combination with copper oxychloride was found to be effective in reducing the Bacterial Blight. The treatments, insecticidal combination with NAA and $\mathrm{MgSO}_{4}$ shown positive results in 
increasing the fruiting branches plant ${ }^{-1}$. All the treatments not shown any phytotoxicity symptom. The insecticide-fungicidal treatments (diafenthiuron 50 WP @0.6 g + carbendazim 50WP @1 g and diafenthiuron 50 WP @0.6 g + copper oxychloride 50WP @ $2 \mathrm{~g}$ ) can be wished in farmer's usage for tackling aphids, leafhoppers, thrips and whiteflies, Alternaria and Blight Bacterial Blight at a given time by reducing number of sprayings and labour cost on sprayings in 90 day old cotton field.

\section{References}

Basavanneppa, M. A., Aladakatti, Y. R., Biradar, D. P., NidagundI, J. M., 2009. Response of Bt cotton to foliar nutrition under irrigated conditions. National Symposium on Bt cotton: Oppurtunities and Prospects, Nagpur, 62.

Bharpoda, T. M., Patel, N. B., Thumar, R. K., Bhatt, N. A., Ghetiya, L. V., Patel, H. C., Borad, P. K., 2014. Evaluation of insecticides against sucking insect pests infesting Bt cotton BG- II. The Bioscan 9(3), 977-980.

Duncan, D. B., 1951. A significance test for differences between ranked treatment means in an analysis of variance. Virginia Journal of Science 2, 171-189.

Gomez, K. A., Gomez, A. A., 1984. Statistical Procedures for Agricultural Research. A Wiley International Science Publication, John Wiley and Sons, New Delhi, 680.
IS, 1973. Indian standard methods of tests for pesticides and their formulations. IS: 6940-1973.

Jagtap, G.P., Jangam, A.M., Dey, U., 2012. Management of bacterial blight of cotton caused by Xanthomonas axonopodis pv. Malvacearum. Scientific Journal of Microbiology 1(1), 10-18.

Rajendran, K., Palchamy, A., Sankaranarayanan, K., Prabakaran, K., Bharathi, K., 2005. Enhancing productivity of summer irrigated cotton through plant growth regulator and foliar nutrition. The Madras Agricultural Journal 98(79), 248-250.

Rajeshwaran, J., Santharam, G., Chandrasekran, S. 2004. Studies on compatibility and phytotoxicity of carbosulfan 25 EC with certain agrochemicals on cotton. Journal of The Entomological Research 28(3), 247252.

Sheo Raj, 1988. Grading for cotton disease, Central Institute of Cotton Research, Nagpur, Bulletin, 1-7.

Stanley, J., Chandrasekaran, S., Preetha, G., Kuttalam, S., 2010. Physical and biological compatibility of diafenthiuron with micro/macro nutrients fungicides and biocontrol agents used in cardamom. Archives of Phytopathology and Plant Protection 43(14), 1396-1406.

\section{How to cite this article:}

Bontha Rajasekar and Mallapur, C.P. 2017. Compatibility of Diafenthiuron with Selected Agro-Chemicals on Bt Cotton. Int.J.Curr.Microbiol.App.Sci. 6(5): 2837-2845. doi: https://doi.org/10.20546/ijcmas.2017.605.321 